Claremont Colleges

Scholarship@ Claremont

All HMC Faculty Publications and Research

HMC Faculty Scholarship

$1-1-2003$

\title{
On the Analysis of Small Displacements of Truss Joints
}

Clive L. Dym

Harvey Mudd College

Harry E. Williams

Harvey Mudd College

\section{Recommended Citation}

Dym, Clive L. and Williams, Harry E., "On the Analysis of Small Displacements of Truss Joints" (2003). All HMC Faculty Publications and Research. Paper 964.

http://scholarship.claremont.edu/hmc_fac_pub/964

This Article - postprint is brought to you for free and open access by the HMC Faculty Scholarship at Scholarship @ Claremont. It has been accepted for inclusion in All HMC Faculty Publications and Research by an authorized administrator of Scholarship @ Claremont. For more information, please contact scholarship@cuc.claremont.edu. 


\title{
ON THE ANALYSIS OF SMALL DISPLACEMENTS OF TRUSS JOINTS
}

\author{
Clive L. Dym and Harry E. Williams \\ Department of Engineering \\ Harvey Mudd College \\ Claremont, CA 91711
}

\section{ABSTRACT}

By emphasizing the kinematic aspects and implications of strain in truss analysis, it is shown that joint displacements can be computed in a straightforward manner.

\section{INTRODUCTION}

A staple of introductory mechanics courses is the analysis of trusses, assemblies of pin-ended bars loaded in uniaxial tension or compression. This is because trusses provide an excellent context in which to introduce free-body diagrams (FBD's), in order to formulate the equations of force equilibrium for each node (pin-joint), and present, in turn, a basis for analyzing more advanced structures. It is important to note that, at this stage, nodal equilibrium equations are written with respect to their undeformed configuration. Furthermore, the examples given are usually statically determinate and lead directly to the determination of the axial forces in each bar.

In contrast, analyses of forces in indeterminate trusses or nodal displacements in determinate trusses are more complex problems. This is largely due to the underlying geometric concept that the axial strain in a bar is the change in length normalized by the original, undeformed length - an essentially nonlinear quantity that can only be determined by analyzing a truss's deformed configuration. Such analyses of the nodal displacements of a truss are often accompanied by complex graphical methods intending to illustrate how (axial) changes in bar lengths produce nodal displacements that are not intuitively evident to a student (or often to their faculty mentors) [1]. Consider, for example, the simple two-bar truss shown in Figure 1. Beginning students may have trouble believing that the node

To appear in the International Journal of Mechanical Engineering Education. 
or joint $B$ moves downward when there is no vertical load (i.e. $P=0$ ). Similarly, it is not obvious to a novice that $B$ should move horizontally if $Q=0$. Compounding this somewhat counterintuitive circumstance are pictures such as Figure 2 that show how such motion occurs as a result, students are told, of both elastic bar extensions and (unextended) rigid body displacements!

It is important to note that when the assumption that the deformed configuration is close to the undeformed configuration-that is, when nodal displacements are small-is justifiable, the problem of evaluating the axial strain in a truss bar becomes linear and solutions for the corresponding forces and displacements are readily obtained. Though this observation is not new, we emphasize it here to promote its use and, consequently, at the same time remove some of the complexities associated with the analysis of indeterminate structures.

We present an alternative approach to elementary truss analysis that is based on a familiar observation: For small displacements of each node, the extension or stretch of each bar is simply the axial component of the relative displacement of the two ends of the bar. This notion clearly builds on Williams' [2] generalized, three-dimensional definitions of the normal engineering strain as being directly calculable from the relative axial displacements of the two ends of a line element. With this observation, the stretch in each bar can be expressed in terms of the components of the displacement of the ends of the bar, and the corresponding force can then be straightforwardly expressed in terms of displacement components by way of a constitutive equation. This process leads to a set of displacement-force equations that, when augmented by the nodal force equilibrium equations, is sufficient to determine the solution for the forces in each bar and the displacements of each node of the truss. We illustrate this process and examine some of its implications below.

We begin by briefly presenting a "classical" advanced analysis of the problem depicted in Figure 1, in order to provide a baseline analysis. Then we establish the strain-relative displacement relation in this context, after which we re-analyze the baseline truss and confirm its results with a nonlinear analysis.

\section{A "Classical" AdVANCEd ANALysis}

For the two-bar truss shown in Figure 1, a standard equilibrium analysis [3] of the joint $B$ produces two equations of equilibrium that can be solved for the axial loads in the two bars. This analysis assumes that the strains are sufficiently small that we can write equilibrium expressions in the undeformed coordinates, which 
gives the two bar forces as:

$$
\begin{aligned}
& F_{1}=F_{A B}=P \tan \theta+Q \\
& F_{2}=F_{B C}=-P / \cos \theta
\end{aligned}
$$

In order to calculate the movement of joint $B$ we can follow a long-established procedure [3] in which we first formulate the complementary energy for the truss, that is,

$$
U^{*}=\sum_{i=1}^{2} \frac{F_{i}^{2} L_{i}}{2 E_{i} A_{i}}
$$

Then, by virtue of Castigliano's second theorem, we can calculate the horizontal and vertical displacements of node $B$ as, respectively:

$$
u=\delta_{\text {Bhoriz }}=\frac{\partial U^{*}}{\partial Q}=\sum_{i=1}^{2} \frac{L_{i} F_{i}}{E_{i} A_{i}} \frac{\partial F_{i}}{\partial Q}=\frac{(P \tan \theta+Q) L_{1}}{E_{1} A_{1}}
$$

and

$$
w=\delta_{\text {Bvert }}=\frac{\partial U^{*}}{\partial P}=\sum_{i=1}^{2} \frac{L_{i} F_{i}}{E_{i} A_{i}} \frac{\partial F_{i}}{\partial P}=\frac{(P \tan \theta+Q) L_{1} \tan \theta}{E_{1} A_{1}}+\frac{P L_{2}}{E_{2} A_{2} \cos ^{2} \theta}
$$

This analysis is straightforward and shows that joint $B$ will move horizontally even in the absence of $Q$ and vertically even when $P=0$. The net movement of node $B$ can also be written as a vector,

$$
\boldsymbol{\delta}_{B}=u \boldsymbol{i}+w \boldsymbol{k}
$$

so that the relative movement of $B$ with respect to the ends of either of the two truss members can here be written as (remembering, of course, that neither of joints $A$ and $C$ moves):

$$
\boldsymbol{\delta}_{B / A}=\boldsymbol{\delta}_{B}-\boldsymbol{\theta}_{A} \quad \boldsymbol{\delta}_{B}
$$

and 


$$
\boldsymbol{\delta}_{B / C}=\boldsymbol{\delta}_{B}-\boldsymbol{\epsilon}_{C} \quad \boldsymbol{\delta}_{B}
$$

While elegant and confirming of our experienced intuition, the foregoing analysis is far too advanced for an introductory course in mechanics or structures.

\section{StRAIN-RELATIVE DISPLACEMENT RELATIONS}

Consider an axially loaded bar that is initially located on the $x$-axis within an orthogonal $(x, y, z)$ frame. If, under load, the bar moves in the $(x, z)$ plane to the new orientation shown in Figure 3, we can define the relative displacement $\boldsymbol{\delta}_{B / A}$ of the point $B$ with respect to the point $A$ much as we just did in eqs. (4) and (5), namely, as

$$
\boldsymbol{\delta}_{B / A}=\boldsymbol{\delta}_{B}-\boldsymbol{\delta}_{A}
$$

where $\boldsymbol{\delta}_{B}$ and $\boldsymbol{\delta}_{A}$ are, respectively, the displacements of the points $B$ and $A$. Furthermore, let the relative displacement have components $u$ and $w$ along the axial $(x)$ and transverse $(z)$ directions, respectively. It readily follows that, when loaded, a bar of initial length $L$ becomes a bar of length $L^{*}$, where:

$$
L^{* 2}=(L+u)^{2}+w^{2}
$$

Hence, for small displacements, for which $(u / L)^{2} \ll<$ and $(w / L)^{2} \ll<$, the bar's new length is:

$$
L^{*} \cong L(1+u / L)
$$

The axial strain $\varepsilon$ in the bar can be expressed in terms of the relative displacement of the bar ends as:

$$
\varepsilon \equiv \frac{\left(L^{*}-L\right)}{L} \cong \frac{u}{L}=\frac{\boldsymbol{\delta}_{B / A} \bullet \boldsymbol{i}}{L}
$$

The strain in a bar can be expressed in terms of its axial force $F$ and its material and geometric properties ( $E$ and $A$ ) of which it is made, as $\varepsilon=F / A E$; using eq. (9), it is clear that the force is: 


$$
F=A E \varepsilon=A E \frac{\left(\boldsymbol{\delta}_{B / A} \bullet \boldsymbol{i}\right)}{L}
$$

Furthermore, if we now introduce the axial $(u)$ and transverse $(w)$ components of the relative displacement of the bar ends (as in eqs. (4) and (5)), the force in the bar (eq. (10)) can be written as:

$$
F=A E \frac{[(u \boldsymbol{i}+w \boldsymbol{k}) \cdot \boldsymbol{i}]}{L}=A E \frac{u}{L}
$$

Equation (11) is, in its final form, a familiar result. However, its intermediate form also admits the introduction of the idea that a joint moves in both axial and transverse directions (relative to any particular bar), even if it is only the axial component of the relative displacement that contributes to that bar's axial force. Furthermore, the net motion of a joint at which several bars meet must be made up of the axial and transverse relative displacement components of all of the bars meeting at that joint. This general, yet comprehensible introduction of joint displacements derives directly from Williams' definition of strain [2].

\section{THE SIMPLE TWO-BAR TRUSS}

Consider the two-bar, pin-ended linkage loaded as shown in Figure 1. An analysis of the forces acting on joint $B$ in the undeformed configuration allows us to find the axial loads in the bars $A B$ and $B C$ as given by eqs. (1). The corresponding displacement of node $B$ is determined by applying eq. (9) to each of the legs in turn. If the displacement of node $B$ is given by eq. (4), we can apply eq. (9) to bar $A B$ to find:

$$
\varepsilon_{1}=\frac{F_{1}}{E_{1} A_{1}}=\frac{\boldsymbol{\delta}_{B / A} \bullet \boldsymbol{e}_{1}}{L_{1}} \equiv \frac{\boldsymbol{\delta}_{B} \bullet \boldsymbol{i}}{L_{1}}=\frac{u}{L_{1}}
$$

For bar $B C$, which has a unit vector along its length equal to,

$$
\boldsymbol{e}_{2}=\sin \theta \boldsymbol{i}-\cos \theta \boldsymbol{k}
$$

we find that strain and relative displacements are related by: 


$$
\varepsilon_{2}=\frac{F_{2}}{E_{2} A_{2}}=\frac{\boldsymbol{\delta}_{B / C} \cdot \boldsymbol{e}_{2}}{L_{2}} \equiv \frac{\boldsymbol{\delta}_{B} \cdot \boldsymbol{e}_{2}}{L_{2}}=\frac{u \sin \theta-w \cos \theta}{L_{2}}
$$

Equations (12) and (14) can be inverted to find joint displacement components in terms of bar strains and, thus, bar forces, that is,

$$
u=\varepsilon_{1} L_{1}=\frac{F_{1} L_{1}}{E_{1} A_{1}}
$$

and

$$
w=\frac{u \sin \theta-\varepsilon_{2} L_{2}}{\cos \theta}=\frac{F_{1} L_{1} \tan \theta}{E_{1} A_{1}}-\frac{F_{2} L_{2}}{E_{2} A_{2} \cos \theta}
$$

Note that the displacement results of eqs. (3) are confirmed when the applied loads from the equilibrium relations (eqs. (1)) are substituted into eqs. (15) for the bar forces.

Consider now the case where only a horizontal load is applied at node $B$, that is, $P=0$. In that case it is easily demonstrated that the two joint displacement components are:

$$
u=\frac{Q L_{1}}{E_{1} A_{1}}
$$

and

$$
w=\frac{Q L_{1} \tan \theta}{E_{1} A_{1}}
$$

Thus, the node $B$ moves an amount represented by the vector:

$$
\boldsymbol{\delta}_{\boldsymbol{B}}=\frac{Q L_{1}}{E_{1} A_{1}}(\boldsymbol{i}+\tan \theta \boldsymbol{k})
$$

The component of the displacement of joint $B$ that lies along the axis of the bar $B C$ can be calculated from the scalar product of eqs. (13) and (17): 


$$
\boldsymbol{e}_{2} \cdot \boldsymbol{\delta}_{\boldsymbol{B}}=\frac{Q L_{1}}{E_{1} A_{1}}(\sin \theta \boldsymbol{i}-\cos \theta \boldsymbol{k}) \cdot(\boldsymbol{i}+\tan \theta \boldsymbol{k})=0
$$

Since the scalar product of eq. (18) vanishes, the displacement of node $B$ must be perpendicular to the bar $B C$ and the force in the bar must vanish (in this linear analysis). This result lends support to the traditional drawings of node displacements mentioned earlier, but the analysis from which it derives provides an explanation and basis for extending the meaning attached to such drawings.

\section{NONLINEAR ANALYSIS OF THE TWO-BAR TRUSS}

A more complete analysis of this truss problem would begin with writing the equations of equilibrium in the deformed configuration (see Figure 3) which, with forces in the deformed state so identified, leads to the result that:

$$
F_{1}^{*} \boldsymbol{e}_{1}^{*}+F_{2}^{*} \boldsymbol{e}_{2}^{*}=Q \boldsymbol{i}+P \boldsymbol{k}
$$

The deformed unit vectors are given by:

$$
\boldsymbol{e}_{1}^{*}=\frac{\left(L_{1} \boldsymbol{i}+\boldsymbol{\delta}\right)}{L_{1}^{*}}, \quad \boldsymbol{e}_{2}^{*}=\frac{\left(L_{2} \boldsymbol{e}_{2}+\boldsymbol{\delta}\right)}{L_{2}^{*}}
$$

where $\boldsymbol{\delta} \equiv \boldsymbol{\delta}_{\boldsymbol{B}}$. Now, eqs. (20) also tell us that the deformed lengths of the bars are given by:

$$
L_{1}^{* 2}=L_{1}^{2}+2 L_{1} \boldsymbol{i} \bullet \boldsymbol{\delta}+\boldsymbol{\delta} \cdot \boldsymbol{\delta} \quad L_{2}^{* 2}=L_{2}^{2}+2 L_{2} \boldsymbol{e}_{2} \cdot \boldsymbol{\delta}+\boldsymbol{\delta} \cdot \boldsymbol{\delta}
$$

so that to first-order accuracy the deformed lengths are:

$$
\begin{aligned}
& \frac{L_{1}^{*}}{L_{1}}=1+\frac{u}{L_{1}}+O\left(\frac{\boldsymbol{\delta} \cdot \boldsymbol{\delta}}{L_{1}^{2}}\right) \cong 1+\varepsilon_{1} \\
& \frac{L_{2}^{*}}{L_{2}}=1+\frac{(u \sin \theta-w \cos \theta)}{L_{2}}+O\left(\frac{\boldsymbol{\delta} \cdot \boldsymbol{\delta}}{L_{2}^{2}}\right) \cong 1+\varepsilon_{2}
\end{aligned}
$$

where we have introduced the linear strains (12) and (13) into eqs. (22) and also used the symbol $O$ to indicate the order of magnitude of terms being neglected. Then, to first order, the unit vectors in the deformed configuration are here: 


$$
\boldsymbol{e}_{i}^{*} \cong \frac{1}{1+\varepsilon_{i}}\left[\boldsymbol{e}_{i}+\frac{\boldsymbol{\delta}}{L_{i}}\right], \quad i=1,2
$$

In view of eq. (4) for the joint displacement vector, the unit vectors are:

$$
\boldsymbol{e}_{1}^{*} \cong \frac{1}{1+\varepsilon_{1}}\left[\left(1+\frac{u}{L_{1}}\right) \boldsymbol{i}+\frac{w}{L_{1}} \boldsymbol{k}\right]
$$

and

$$
\boldsymbol{e}_{2}^{*} \cong \frac{1}{1+\varepsilon_{2}}\left[\left(\sin \theta+\frac{u}{L_{2}}\right) \boldsymbol{i}+\left(-\cos \theta+\frac{w}{L_{2}}\right) \boldsymbol{k}\right]
$$

Equations (24) can be used to write the equilibrium conditions in the deformedstate (19) as:

$$
\frac{F_{1}^{*}}{1+\varepsilon_{1}}\left[\left(1+\frac{u}{L_{1}}\right) \boldsymbol{i}+\frac{w}{L_{1}} \boldsymbol{k}\right]+\frac{F_{2}^{*}}{1+\varepsilon_{2}}\left[\left(\sin \theta+\frac{u}{L_{2}}\right) \boldsymbol{i}+\left(-\cos \theta+\frac{w}{L_{2}}\right) \boldsymbol{k}\right]=Q \boldsymbol{i}+P \boldsymbol{k}
$$

The vector equation (25) represents equilibrium in the $x$ - and $z$-directions, whose individual scalar components can be extracted with appropriate scalar products:

$$
F_{1}^{*}\left(1+\varepsilon_{2}\right)\left(1+\frac{u}{L_{1}}\right)+F_{2}^{*}\left(1+\varepsilon_{1}\right)\left(\sin \theta+\frac{u}{L_{2}}\right)=Q\left(1+\varepsilon_{1}\right)\left(1+\varepsilon_{2}\right)
$$

and

$$
F_{1}^{*}\left(1+\varepsilon_{2}\right) \frac{w}{L_{1}}+F_{2}^{*}\left(1+\varepsilon_{1}\right)\left(-\cos \theta+\frac{w}{L_{2}}\right)=P\left(1+\varepsilon_{1}\right)\left(1+\varepsilon_{2}\right)
$$

Equations (26) can be straightforwardly solved for the bar forces in the deformed state to first-order accuracy, and those bar forces are:

$$
F_{1}^{*} \cong Q+P \tan \theta+\varepsilon_{1}\left(P \tan \theta-Q \tan ^{2} \theta\right)+\varepsilon_{2}\left(\frac{Q}{\cos ^{2} \theta}\right)
$$


and

$$
F_{2}^{*} \cong-\frac{P}{\cos \theta}+\varepsilon_{1}\left(\frac{Q \sin \theta}{\cos ^{2} \theta}\right)-\varepsilon_{2}\left(\frac{P}{\cos \theta}+\frac{Q}{\sin \theta \cos ^{2} \theta}\right)
$$

Using eqs. (1), (12), and (14), we can also write these results as:

$$
F_{1}^{*} \cong F_{1}+\frac{F_{1}}{E_{1} A_{1}}\left(P \tan \theta-Q \tan ^{2} \theta\right)+\frac{F_{2}}{E_{2} A_{2}}\left(\frac{Q}{\cos ^{2} \theta}\right)
$$

and

$$
F_{2}^{*} \cong F_{2}+\frac{F_{1}}{E_{1} A_{1}}\left(\frac{Q \sin \theta}{\cos ^{2} \theta}\right)-\frac{F_{2}}{E_{2} A_{2}}\left(\frac{P}{\cos \theta}+\frac{Q}{\sin \theta \cos ^{2} \theta}\right)
$$

Not surprisingly, eqs. (28) confirm the zeroth-order results given in eqs. (1), and that the corrections resulting from a nonlinear analysis are comfortably negligible as long as the strains remain small. In addition, eqs. (28) help us articulate a clear explanation of how a horizontal load produces a non-zero bar force in the unloaded bar $B C$. For that case, which brought us to this analysis in the first place, $P=0$, the sole external load is the horizontal force $Q$, and the linear bar forces are:

$$
F_{1}=Q, \quad F_{2}=0
$$

From eqs. (28), the first-order, nonlinear bar forces are:

$$
F_{1}^{*} \cong Q-\frac{Q^{2}}{E_{1} A_{1}} \tan ^{2} \theta=Q\left(1+O\left(\varepsilon_{1}\right)\right)
$$

and

$$
F_{2}^{*} \cong 0+\frac{Q^{2}}{E_{1} A_{1}}\left(\frac{\sin \theta}{\cos ^{2} \theta}\right)=Q O\left(\varepsilon_{1}\right)
$$

So, referring back to the pedagogical theme of this paper, while the foregoing 
nonlinear analysis is clearly beyond what one would expect to discuss in an introductory course, it certainly lays a foundation for presenting the results (30), accompanied by remarks about the limitations that often attend linear analyses.

\section{CONCLUSIONS}

In this note we have endeavored to show how the kinematic aspects of axial strain can be used as a vehicle for calculating and explaining joint movements in trusses. The particular truss problem illustrated here is a simple one that, nonetheless, is a source of confusion for novice students because some of its results are, for them, not intuitively evident. Now, a more intuitive and more consistent approach to the calculation and interpretation of truss joint movement is enabled by Williams' characterization of strain.

\section{REFERENCES}

1. W. A. Nash, Strength of Materials, 4th ed., McGraw-Hill, New York, 1998.

2. H. E. Williams, "On Introducing Engineering Strain," International Journal of Mechanical Engineering Education, 29/4 (October 2001), pp. 397-403.

3. C. L. Dym, Structural Modeling and Analysis, Cambridge University Press, New York, 1997.

\section{ACKNOWLEDGEMENT}

The authors are grateful to an anonymous reviewer for providing a very thorough review of an earlier draft of this paper.

\section{LIST OF FIGURES}

Figure 1. A simple two-bar truss carrying vertical and horizontal loads.

Figure 2. A (greatly exaggerated) geometric depiction of one explanation for the movement of the free node in the two-bar truss of Figure 1. The node displacement is found by erecting a normal to each bar extension, $\boldsymbol{\delta}_{1}$ and $\boldsymbol{\delta}_{2}$, thus approximating the "rigid body" displacements tangent to circular arcs drawn through each bar's pinned end.

Figure 3. The (greatly exaggerated) deformed configuration of the simple twobar truss of Figure 1. 


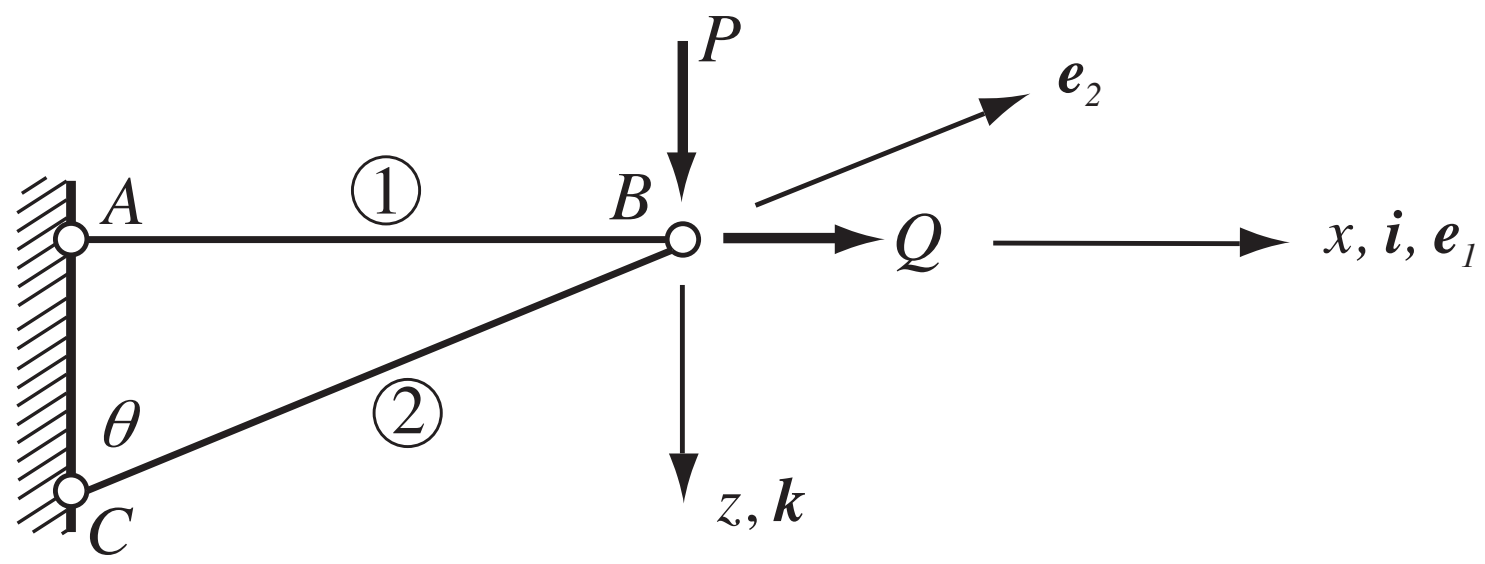

Figure 1. A simple two-bar truss carrying vertical and horizontal loads. 


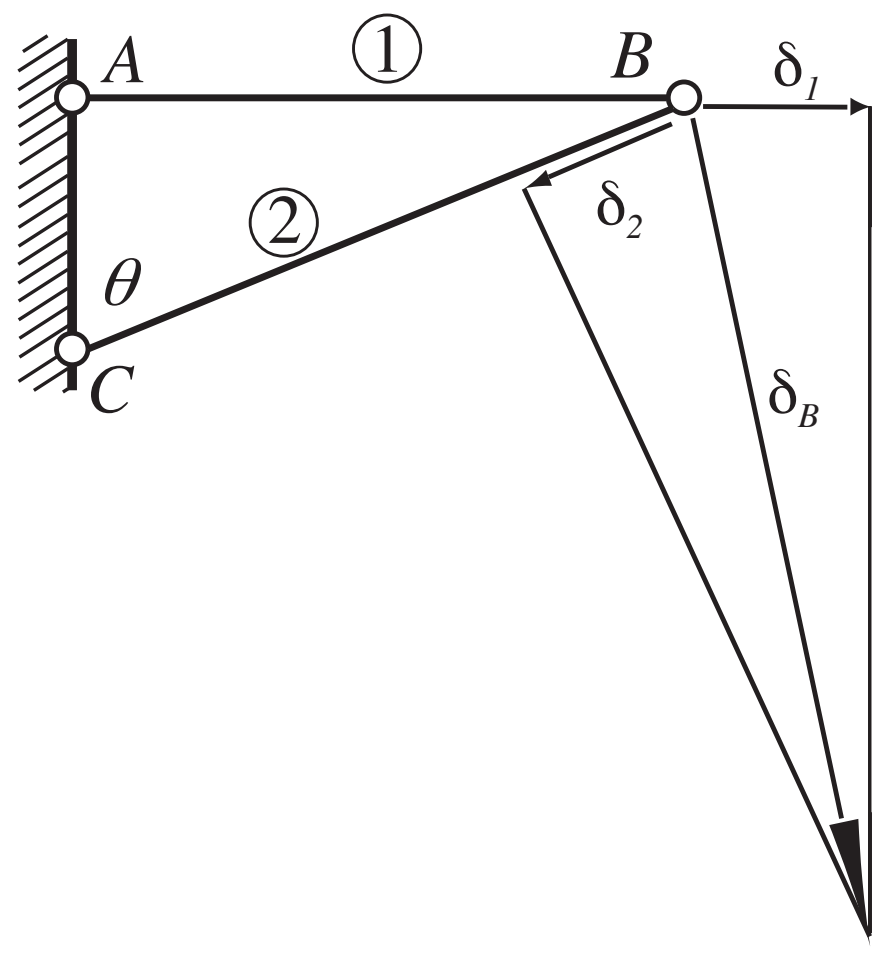

Figure 2. A (greatly exaggerated) geometric depiction of one explanation for the movement of the free node in the two-bar truss of Figure 1. The node displacement is found by erecting a normal to each bar extension, $\boldsymbol{\delta}_{1}$ and $\boldsymbol{\delta}_{2}$, thus approximating the "rigid body" displacements tangent to circular arcs drawn through each bar's pinned end. 


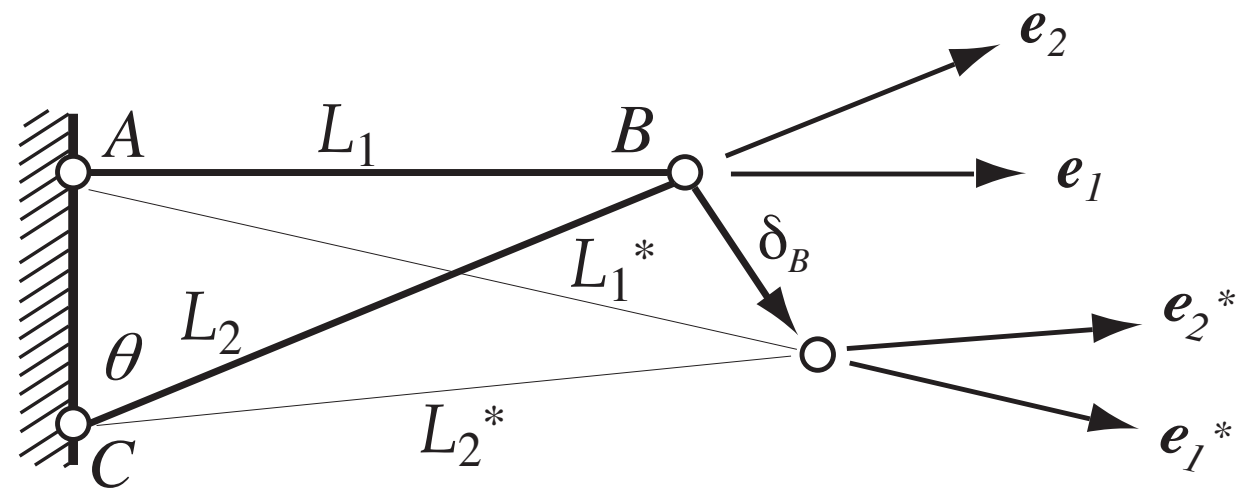

Figure 3. The (greatly exaggerated) deformed configuration of the simple twobar truss of Figure 1. 